“C 2017 IEEE. Personal use of this material is permitted. Permission from IEEE must be obtained for all other uses, in any current or future media, including reprinting/republishing this material for advertising or promotional purposes, creating new collective works, for resale or redistribution to servers or lists, or reuse of any copyrighted component of this work in other works." 


\title{
Spatio-temporal Environmental Monitoring for Smart Buildings*
}

\author{
Linh Nguyen ${ }^{1}$, Guoqiang $\mathrm{Hu}^{1}$ and Costas J. Spanos ${ }^{2}$
}

\begin{abstract}
The paper addresses the problem of efficiently monitoring environmental fields in a smart building by the use of a network of wireless noisy sensors that take discretelypredefined measurements at their locations through time. It is proposed that the indoor environmental fields are statistically modeled by spatio-temporal non-parametric Gaussian processes. The proposed models are able to effectively predict and estimate the indoor climate parameters at any time and at any locations of interest, which can be utilized to create timely maps of indoor environments. More importantly, the monitoring results are practically crucial for building management systems to efficiently control energy consumption and maximally improve human comfort in the building. The proposed approach was implemented in a real tested space in a university building, where the obtained results are highly promising.
\end{abstract}

\section{INTRODUCTION}

Recent researches have shown that energy consumption in residential and commercial buildings is about $40 \%$ of total energy consumed in the entire world [1]. Particularly, heating, ventilating, and air conditioning (HVAC) systems account for up to $47 \%$ of the building energy [2]. Nevertheless, it is also reported that at least $30 \%$ of the electric energy consumed by the HVAC systems are wasted [3]. On the other hand, there are more and more demands on standards for indoor environmental qualities in building spaces [4]. That is, indoor climates substantially impact on well-being and productivity of the building inhabitants. Therefore, reducing the energy consumption and increasing the human comfort are paramount to economically and socially justify smart buildings. While the human comfort indexes are based on the indoor spatial fields such as air temperature, relative humidity, and carbon dioxide, it is required to optimally control the indoor environments. To this end, deeply understanding of building environments is essential for efficient building energy managements. Recent attentions consist of observing indoor environmental parameters in secondary schools' classrooms [5] and in university buildings [6], monitoring room temperature in offices [7], and detecting fire in buildings [8]. However, to the best of our knowledge, in all aforementioned existing works, authors have only concerned about locally monitoring the indoor spatial parameters at some specific points. Mapping environmental fields in the whole building space is still questionable.

*This work was supported by the Building and Construction Authority of Singapore under EIRP-BEE Grant NRF2013EWT-EIRP004-051.

${ }^{1}$ School of Electrical and Electronic Engineering, Nanyang Technological University, Singapore linh.v.nguyen@ieee.org, gqhulantu.edu.sg

${ }^{2}$ Department of Electrical Engineering and Computer Science, University of California, Berkeley, CA 94720, United States spanos@berkeley.edu
The existing approaches mainly rely on the mathematically analytical models such as partial differential or NavierStokes equations [9]. The premise behind these heat transfer models consists of incorporating architectural parameters of the building into the models and presenting the most comprehensive description of the thermal processes in the building in the heat transfer equations. Notwithstanding, in order to let the aforementioned models be computable, all complexity of thermal interactions, unmeasured disturbances, and uncertainties in thermal properties of structural elements is simplified by various assumptions. Moreover, requirements of the analytical models to be known a priori make them more challenging to be more reliable [10].

Therefore, in this paper, a spatio-temporal model based on the non-parametric data-driven Gaussian processes (GP) is proposed to statistically represent distribution of the environmental field in the building space. In fact, all parameters of the GP models can be directly learned from measurements collected by a wireless sensor network, then it can fully delineate distributions of the spatial fields. The main advantages of the proposed model are able to efficiently temporally estimate and predict the indoor spatial fields in the rest of the building space in which there are no measurements conducted. This leads to a fact that a map/surface of the interested environmental parameter in the building can be created and sent out to the building management systems as a timely feedback. Based on information obtained in the environmental map, controlling laws/strategies can be adjusted to regulate the indoor climate to reach to expected values.

The paper is organized as follows. A spatio-temporal model for environmental monitoring is presented in Section II. Section III discusses results in the real scenarios conducted in the experimental spaces. Finally, conclusions of the work are provided in Section IV.

\section{SPATIO-TEMPORAL MOdel FOR INDOOR ENVIRONMENT}

Let $\mathbb{R}$ denote the set of real numbers. Boldface case letters are defined as vectors or matrices. The norm of a vector in the Euclidean space is also denoted by $\|\cdot\|$. We let $\mathbb{E}$ define the expectation operator while $|\cdot|$ defines the absolute value of a scalar. The transpose of a matrix $A$ is denoted as $A^{T}$. Other notations will be explained as and when they occur.

\section{A. A Model of Spatio-temporal Fields}

We consider a network of $n$ wireless sensors that are spatially deployed in buildings to observe indoor environmental fields such as temperature, relative humidity and carbon 
dioxide. Each sensor take discrete measurements over time at predefined instants; then the data is wirelessly transmitted to a base station (or sink) via a routing tree.

In the indoor space of interest $\mathcal{Q} \subset \mathbb{R}^{d}$, let spatial locations of the wireless sensors within $\mathcal{Q}$ denote as $\mathbf{s}=$ $\left(\mathbf{s}_{1}^{T}, \mathbf{s}_{2}^{T}, \cdots, \mathbf{s}_{n}^{T}\right)^{T} \in \mathbb{R}^{d \times n}$. In this study, we suppose that at each of collecting instants, all wireless sensors measure indoor environments concurrently. Hence, we define the time at which sensors take measurements as $\mathbf{t}=\left(t_{1}, t_{2}, \cdots, t_{m}\right)^{T} \in$ $\mathbb{R}^{m}$. As a result, a spatio-temporal location can be represented as a point on $\mathbb{R}^{d} \times \mathbb{R}$. Now, the measurement of a sensor at a location $\mathbf{s}_{i}$ and at time $t_{j}$ can be specified by

$$
y\left(\mathbf{s}_{i}, t_{j}\right)=z\left(\mathbf{s}_{i}, t_{j}\right)+\varepsilon\left(\mathbf{s}_{i}, t_{j}\right),
$$

where $z\left(\mathbf{s}_{i}, t_{j}\right)$ and $\varepsilon\left(\mathbf{s}_{i}, t_{j}\right)$ are a random variable and a noise at a spatio-temporal location $\left(\mathbf{s}_{i}, t_{j}\right), i=1, \cdots, n$ and $j=1, \cdots, m$. The noise is assumed to be independent and identically distributed. $\varepsilon\left(\mathbf{s}_{i}, t_{j}\right)$ is normally distributed with a zero mean and an unknown variance $\tau^{2}$. Therefore, if $Y(\mathbf{s}, \mathbf{t})=\left(y\left(\mathbf{s}_{1}, t_{1}\right), \cdots, y\left(\mathbf{s}_{n}, t_{m}\right)\right)^{T} \in \mathbb{R}^{n \times m}$ denotes a vector of collective measurements gathered by the network of wireless sensors up to current time $t_{m}$, then it can be delineated by

$$
Y(\mathbf{s}, \mathbf{t})=Z(\mathbf{s}, \mathbf{t})+\epsilon(\mathbf{s}, \mathbf{t}),
$$

where $\epsilon(\mathbf{s}, \mathbf{t})=\left(\varepsilon\left(\mathbf{s}_{1}, t_{1}\right), \cdots, \varepsilon\left(\mathbf{s}_{n}, t_{m}\right)\right)^{T} \in \mathbb{R}^{n \times m}$, and $Z(\mathbf{s}, \mathbf{t})=\left(z\left(\mathbf{s}_{1}, t_{1}\right), \cdots, z\left(\mathbf{s}_{n}, t_{m}\right)\right)^{T} \in \mathbb{R}^{n \times m}$ is a spatiotemporal random field that is proposed to be followed a multivariate Gaussian distribution. The mean of the spatiotemporal $Z(\mathbf{s}, \mathbf{t})$ is defined by $\mu(\mathbf{s}, \mathbf{t})=\mathbb{E}[Z(\mathbf{s}, \mathbf{t})]$, and each element of its covariance matrix can be computed by a covariance function as specified

$$
\operatorname{cov}\left(\left(\mathbf{s}_{i}, t_{j}\right),\left(\mathbf{s}_{k}, t_{l}\right)\right)=\sigma^{2} \operatorname{corr}\left(z\left(\mathbf{s}_{i}, t_{j}\right), z\left(\mathbf{s}_{k}, t_{l}\right)\right),
$$

for any two pairs of spatio-temporal locations $\left(\mathbf{s}_{i}, t_{j}\right)$ and $\left(\mathbf{s}_{k}, t_{l}\right)$ on $\mathbb{R}^{d} \times \mathbb{R}, k=1, \cdots, n$ and $l=1, \cdots, m$. Here, $\sigma^{2}$ is a marginal variance, and $\operatorname{corr}\left(z\left(\mathbf{s}_{i}, t_{j}\right), z\left(\mathbf{s}_{k}, t_{l}\right)\right)$ is a correlation function based on two random variables $z\left(\mathbf{s}_{i}, t_{j}\right)$ and $z\left(\mathbf{s}_{k}, t_{l}\right)$.

Notwithstanding, in fact, the main challenges in analyzing the spatio-temporal environmental fields is how to choose a correlation function that can best present the spatiotemporal dependence of the observations. In recent research, there are discussions about types of correlation functions for space-time models [11]. There are two major sorts of the spatio-temporal correlation models that are separable and non-separable. Theoretically, the spatio-temporal nonseparable correlation models have been thought to better capture possible space-time interactions. Nevertheless, due to computational complexity of the spatio-temporal nonseparable correlation functions, especially when applied in large data sets, in this work, we choose spatio-temporal separable correlation models. One of frequently used spacetime separable correlation functions is the double-stable model, as given by

$\operatorname{corr}(\mathbf{h}, u)=\exp \left(-\left(\frac{\left\|\mathbf{s}_{i}-\mathbf{s}_{k}\right\|}{\psi_{s}}\right)^{\kappa_{s}}-\left(\frac{\left|t_{j}-t_{l}\right|}{\psi_{t}}\right)^{\kappa_{t}}\right)$,

where $\psi_{s}$ and $\psi_{t}$ are two positive scale parameters in terms of space and time, respectively. $\psi_{s}$ and $\psi_{t}$ are referred to as reduction rates of the dependence between two random variables $z\left(\mathbf{s}_{i}, t_{j}\right)$ and $z\left(\mathbf{s}_{k}, t_{l}\right)$ at two spatio-temporal locations $\left(\mathbf{s}_{i}, t_{j}\right)$ and $\left(\mathbf{s}_{k}, t_{l}\right)$ when $\left\|\mathbf{s}_{i}-\mathbf{s}_{k}\right\|$ or $\left|t_{j}-t_{l}\right|$ increase. $\kappa_{s}$ and $\kappa_{t}$ are smoothing parameters, $0<\kappa_{s}, \kappa_{t} \leqslant 2$. In practice, $\kappa_{s}$ and $\kappa_{t}$ can be chosen a priori. Specifically, if $\kappa_{s}=1$ and $\kappa_{t}=1$, we have an exponential space-time separable correlation function. Similarly, setting $\kappa_{s}=2$ and $\kappa_{t}=2$ leads to a Gaussian space-time separable correlation model. It can be seen that in (4) the correlation function only depends on $\left\|\mathbf{s}_{i}-\mathbf{s}_{k}\right\|$ and $\left|t_{j}-t_{l}\right|$, it is called spatially temporally isotropic. Note that the parameters $\sigma^{2}, \tau^{2}, \psi_{s}$ and $\psi_{t}$ are unknown but can be learned by the use of all available measurements.

\section{B. Spatio-temporal Predictive Inference}

From (2), it is derived that

$$
Y(\mathbf{s}, \mathbf{t}) \sim \mathcal{N}\left(\mu, \Sigma+\tau^{2} I\right),
$$

where $\mu$ is a scalar value that is a mean of all available observations. $\Sigma$ is the $n m \times n m$ covariance matrix of $Y(\mathbf{s}, \mathbf{t})$, where its elements can be computed by using (3). $I$ is a $n m \times n m$ identity matrix. Let us first define $\mathbf{s}^{*}$ and $\mathbf{t}^{*}$ as a matrix of coordinates of all unmeasured locations of interest and a vector of specific instants when we would monitor the environments, respectively. We also denote $Z^{*}\left(\mathbf{s}^{*}, \mathbf{t}^{*}\right)$ is a vector of random variables at locations $\mathbf{s}^{*}$ and at times $\mathbf{t}^{*}$. Note that $Y(\mathbf{s}, \mathbf{t})$ and $Z^{*}\left(\mathbf{s}^{*}, \mathbf{t}^{*}\right)$ are variables in the same space $\mathcal{Q}$, though they may not be observed at the same times. Hence, the joint distribution of $Y(\mathbf{s}, \mathbf{t})$ and $Z^{*}\left(\mathbf{s}^{*}, \mathbf{t}^{*}\right)$ is specified by

$$
\left[\begin{array}{c}
Y(\mathbf{s}, \mathbf{t}) \\
Z^{*}\left(\mathbf{s}^{*}, \mathbf{t}^{*}\right)
\end{array}\right] \sim \mathcal{N}\left(\left[\begin{array}{l}
\mu \\
\mu_{*}
\end{array}\right],\left[\begin{array}{cc}
\Sigma+\tau^{2} I & \Sigma_{*} \\
\Sigma_{*}^{T} & \Sigma_{* *}
\end{array}\right]\right),
$$

where $\mu_{*}$ and $\Sigma_{* *}$ are the mean and the covariance matrix of $Z^{*}\left(\mathbf{s}^{*}, \mathbf{t}^{*}\right)$. In this scenario, we suppose that the mean is consistent, $\mu_{*}=\mu$. Each element of the covariance matrix $\Sigma_{* *}$ is computed by the covariance function $\operatorname{cov}\left(\left(\mathbf{s}_{i}^{*}, t_{j}^{*}\right),\left(\mathbf{s}_{k}^{*}, t_{l}^{*}\right)\right)$ as given in (3). Notice that $\mathbf{s}_{i}^{*}, \mathbf{s}_{k}^{*} \in \mathbf{s}^{*}$ and $t_{j}^{*}, t_{l}^{*} \in \mathbf{t}^{*}$. In addition, $\Sigma_{*}$ is the cross-covariance matrix representing the dependence between $Y(\mathbf{s}, \mathbf{t})$ and $Z^{*}\left(\mathbf{s}^{*}, \mathbf{t}^{*}\right)$, whose elements are calculated by the crosscovariance function $\operatorname{cov}\left(\left(\mathbf{s}_{i}, t_{j}\right),\left(\mathbf{s}_{k}^{*}, t_{l}^{*}\right)\right)$. From (6), we can now infer the posterior distribution of $Z^{*}\left(\mathbf{s}^{*}, \mathbf{t}^{*}\right)$, given the measurements $Y(\mathbf{s}, \mathbf{t})$, by taking the following form.

$$
Z^{*}\left(\mathbf{s}^{*}, \mathbf{t}^{*}\right) \mid Y(\mathbf{s}, \mathbf{t}) \sim \mathcal{N}\left(\mu_{* \mid Y(\mathbf{s}, \mathbf{t})}, \Sigma_{* * \mid Y(\mathbf{s}, \mathbf{t})}\right),
$$

where

$$
\begin{gathered}
\mu_{* \mid Y(\mathbf{s}, \mathbf{t})}=\mu_{*}+\Sigma_{*}^{T}\left(\Sigma+\tau^{2} I\right)^{-1}(Y(\mathbf{s}, \mathbf{t})-\mu), \\
\Sigma_{* * \mid Y(\mathbf{s}, \mathbf{t})}=\Sigma_{* *}-\Sigma_{*}^{T}\left(\Sigma+\tau^{2} I\right)^{-1} \Sigma_{*} .
\end{gathered}
$$




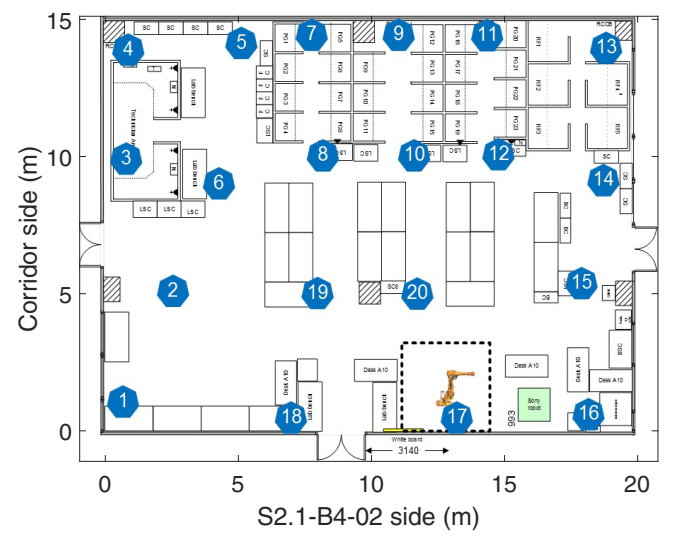

Fig. 1: Random sensor deployments at beginning in S2.1B4-01 room, Nanyang Technological University

It can be clearly seen that the indoor environmental fields at any unobserved locations can be predicted at any time by only using available measurements. In order to demonstrate the effectiveness of the approach of predicting spatiotemporal environmental fields, we implement and discuss the method in the real scenarios in the following section.

\section{REAL EXPERIMENTS IN BUILDINGS}

\section{A. Indoor Experimental Description}

In order to demonstrate effectiveness of the spatiotemporal model in intelligently monitoring indoor environmental fields, we conducted experiments at the S2.1-B401 room in the Nanyang Technological University campus, Singapore. The experiments were carried out during the time from 7 March to 10 April 2016. The experimented room is sized $19.80 \mathrm{~m}$ in the length and $14.86 \mathrm{~m}$ in the width. A brief snapshot of the room can be seen in the Fig. 1 . Better understanding the room configuration leads to better analysis of the environmental variations. Thus, arrangements of the S2.1-B4-01 room are delineated as the following. The room has 3 doors. Opening and closing these doors obviously affect on indoor environments. The main door is in the corridor side, which room occupants usually use for their entrance and exit of the room. The back door next to the sensor nodes 14 and 15 is sometimes utilized in some particular circumstances. And the door next to the sensor node 18 is completely closed at all time. From the main door, the left hand side of the room is the area where there have cubicles with desk-work stations for research staff and research students. When one enters the room, it can be seen there is a technical area in the first left corner in which servers and technical facilities are located. In the middle of the room, there are four rows of tables with personal computers for undergraduate classes. In addition, in the far right corner, there have robots with equipment for other research experiments. On the ceiling, it has 16 air inlets and 16 air outlets, respectively. The air inlets supplied to the room conditioned air with specific levels of temperature, relative humidity and carbon dioxide, which are expected to be similar to those of fresh air. And exhausted air gets out the room via air outlets. Note that due to management reasons, there are some air inlets and outlets to be off during the time the experiments were conducted.

In the context of occupancy, there are daily 28 research staff and research students working in the room in weekdays; some of them are still in on weekends. Moreover, it usually has about 20 undergraduate students attending their experimental classes in this room. Notice that occupants' activities considerably influence on the indoor environmental processes.

In the experiments, we utilized two wireless networks of 10 Libelium temperature sensors and 10 Monnit temperature sensors. These sensor nodes, after measuring indoor temperatures, send the measurements directly to the network routers via a one-hop routing structure. The data can be accessed from any internet connected devices. The downloaded measurements were then employed to train and validate the space-time models of the environmental fields in the room.

To provide good feedback for strategies of controlling indoor environments, which is aimed to increase human comfort, in this work we positioned all sensors at sitting levels. 20 sensors measuring spatio-temporal temperatures in the room were deliberately located and numbered as shown in Fig. 1.

\section{B. Result Discussions}

The temperature measurements were gathered in 5 week time. The first four week collective data was used for training space-time models; and the last week sensor readings were utilized to evaluate the learned models. Note that the sensors were set to take environmental measurements every 2 hours. That is, each sensor could gather 84 measurements at its location every week.

Nonetheless, in the first presentation, we discuss real variations of spatial processes over time at a sensor node location. For instance, realistic temperatures in the tested room through 4 week time, from 7 March to 3 April 2016, at 4 specific sensor locations are demonstrated in Fig. 2. The four chosen sensor positions represent the four particular areas in the room. Specifically, the node 6 is close to the door, the node 13 is in the cubicle area and next to a corner, the node 17 is in the area with most nonhuman activities, and the node 20 is in the middle of the room. The measured temperatures are graphically curved into weekly forms. Thus, it is interesting that we can also compare location-self temperatures among weeks.

In general, temperature in the experimented room is iteratively high during the daytime and low during the nighttime, except that at a location of the sensor node 17. Recordings of the temperature sensor 17 in the four week experiments are fluctuated in a non-ordered way, though their variations are small, ranging from $22.2^{\circ} \mathrm{C}$ to $23.2^{\circ} \mathrm{C}$. The reasons are that there were almost no human activities and that air inlets and outlets in an area around the sensor 17 were off during experimental time. More importantly, the temperature in the room at the selected locations was ranged from $21.4^{\circ} \mathrm{C}$ to 


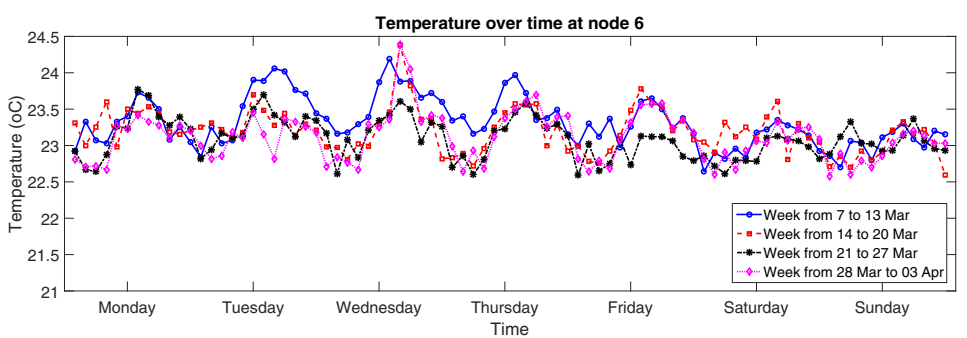

(a)

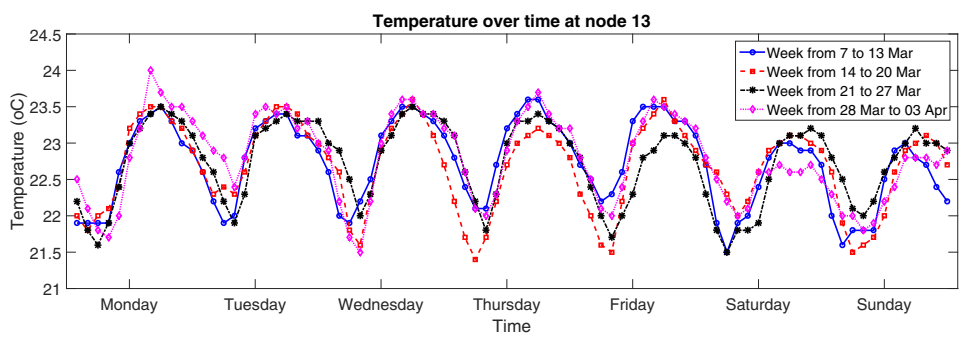

(b)

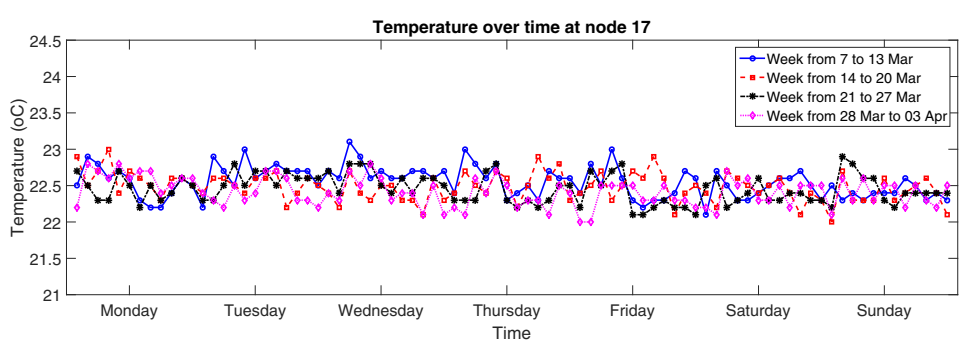

(c)

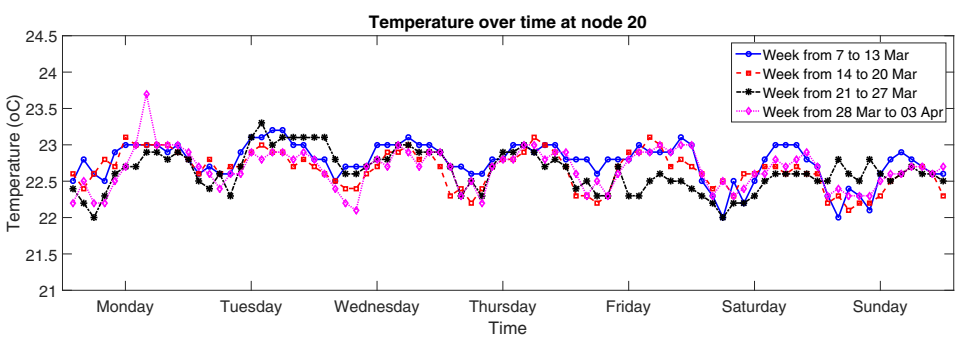

(d)

Fig. 2: Real temperature observations over time at sensor nodes in weeks from 7 March to 3 April 2016 in S2.1-B4-01 room, Nanyang Technological University: (a) node 6, (b) node 13, (c) node 17, (d) node 20. Refer to Fig. 1 for their locations.

$24.2^{\circ} \mathrm{C}$. In fact, this temperature is always lower than that is outside since Singapore is a tropical rain-forest country whose climate is with year-round hot temperature. It can be obviously seen that the temperature at any time on weekends is always lower than that is on weekdays. However, the Friday 25 March, a public holiday in Singapore, was cooler than the other weekdays. On the other hand, the heat inside the room daily gets a peak around 2:00pm to $4: 00 \mathrm{pm}$ and then cools down to the lowest value at approximate 4:00am to 6:00am.

During daytime the cubicle area is often hotter than the others, which can be seen in curves of the temperatures measured at the sensor node 13 , a representative position in the cubicle area, though it really cool in nighttime. This phenomenon can be interpreted by the occupant activities and the heat exhausted from working personal computers. Notwithstanding, even though there are no heat-exhausted facilities around the node 6 , the temperature captured by the sensor 6 is still high as compared with that in the cubicle area. This high temperature was caused by frequently-opened door activities.

In the following we discuss about maps of the environmental fields in the room. Of the collected dataset, first four week temperature measurements were employed to learn a spatio-temporal model, which is introduced in Section II. For instance, we utilized 6720 temperature values collected by 20 sensors over 4 weeks to develop a space-time model that comprehensively represents for the temperature distribution 

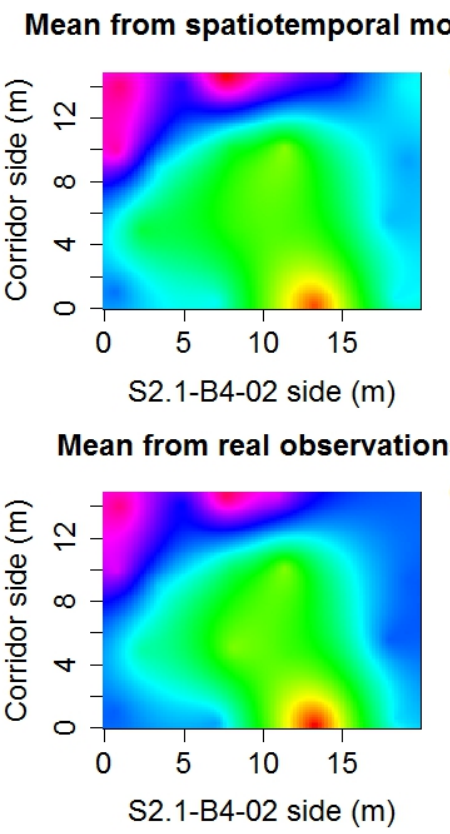

(a)

\section{Std error from spatiotemporal model}

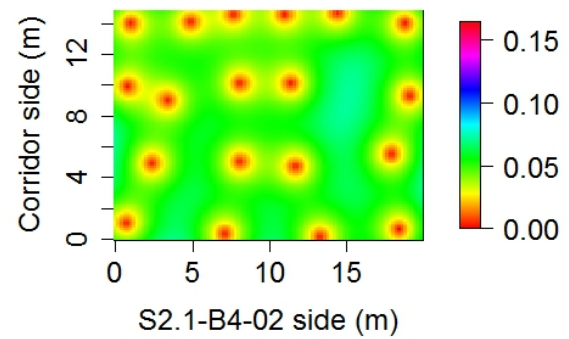

Std error from real obervations

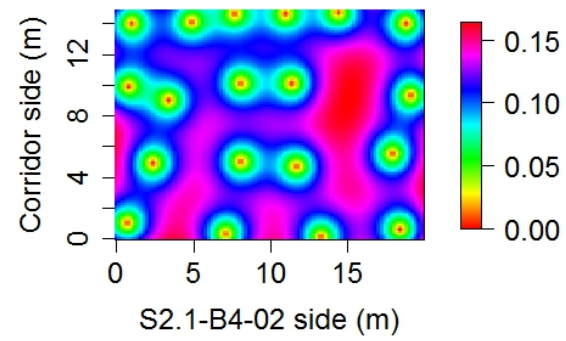

(d)
Mean from spatiotemporal model

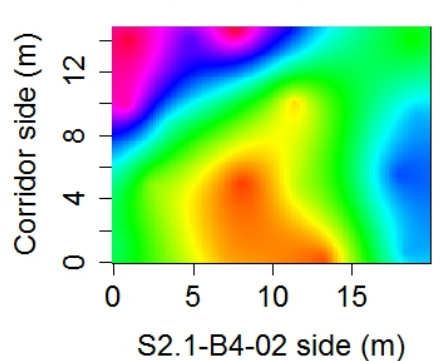

Mean from real observations

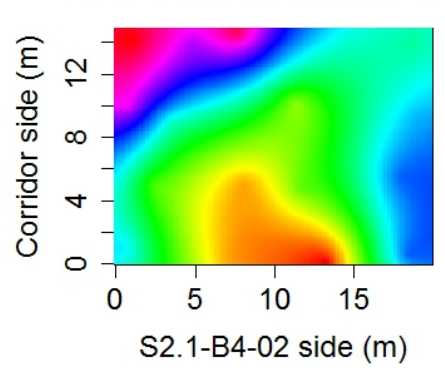

(b)

\section{Std error from spatiotemporal model}

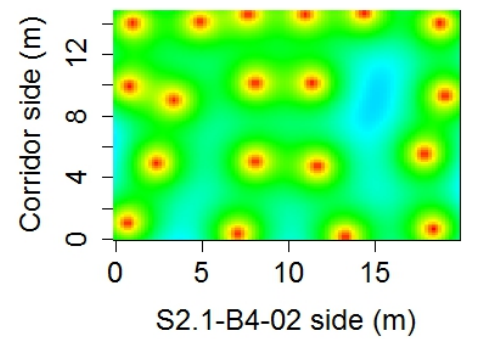

Std error from real obervations

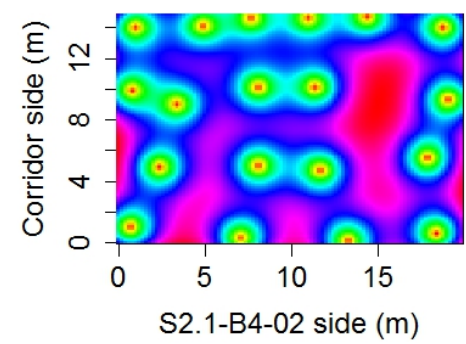

(e)

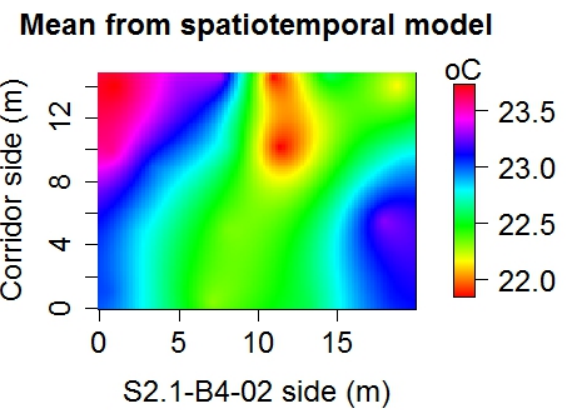

Mean from real observations

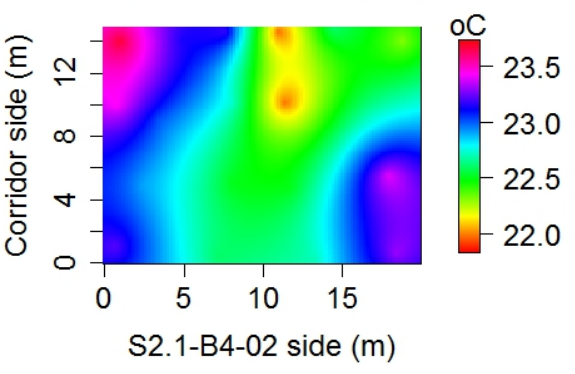

(c)

\section{Std error from spatiotemporal model}

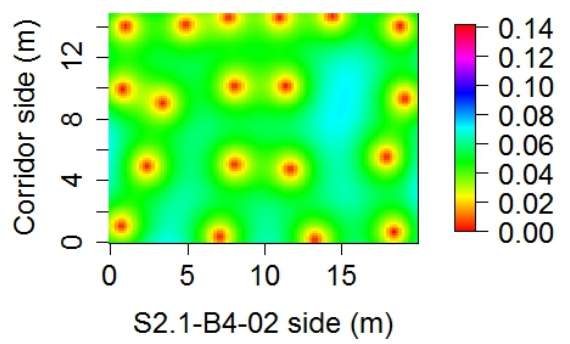

Std error from real obervations

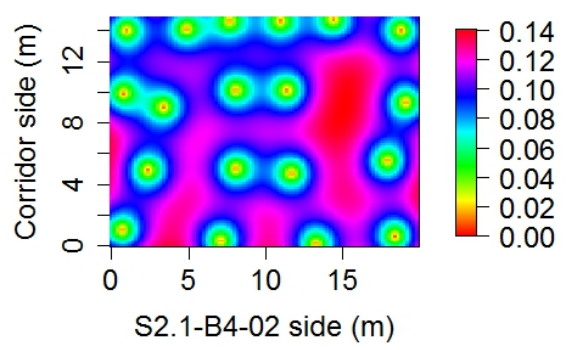

(f)

Fig. 3: Predicted temperature fields in S2.1-B4-01 room, Nanyang Technological University at different times on different days: (a) at 16:45 on 7 April 2016, (b) at 10:50 on 8 April 2016 and (c) at 00:45 on 10 April 2016. Corresponding prediction standard errors are also presented: (d) at 16:45 on 7 April 2016, (e) at 10:50 on 8 April 2016 and (f) at 00:45 on 10 April 2016. Ranges of the fields and the standard errors are illustrated in color bars. 
in the tested room at any time. We call this model as model $_{1}$. The learned model of the temperature in the room was then used to predict the temperature field at any locations in the room at any time. In equivalent words, we can totally create a highly accurate map of the heat in the whole room at any time during the week. To demonstrate this fact, the trained spatio-temporal temperature model was employed to predict the maps of the heat in the experimented room at three different times, 10:50am in the morning, 4:45pm in the afternoon and 0:45am in the night in the evaluating week, from 4 to 10 April 2016, shown in Fig. 3. The demonstrations were conducted in two weekdays, Thursday 7 April and Friday 8 April 2016, and one weekend, 10 April 2016. For the purpose of comparisons, our sensors also measured the room temperature at these three different times. A purely spatial model [12] was learned by the use of each set of data gathered at each tested time. We call this model as model $_{2}^{t}$. Since model $_{2}^{t}$ is purely spatial, at every time $t$ we have one $\operatorname{model}_{2}^{t}$ learned separately. Each learned spatial model model $_{2}^{t}$ was then employed to predict the heat map of the whole room at the corresponding time $t$. The results are also illustrated in Fig. 3.

Figures $3 \mathrm{a}, 3 \mathrm{~b}$ and $3 \mathrm{c}$ illustrate the predicted temperature fields in the whole tested room at 16:45 on Thursday 7 April, 10:50 on Friday 8 April and 00:45 on Sunday 10 April 2016, respectively. In each of these sub-figures, there are two mean heat maps arranged in a column of which the top demonstrates the predicted field obtained by the learned spatio-temporal model model $_{1}$ and the bottom shows the estimated field obtained by model $_{2}^{t}$ that is directly learned by the realistic measurements at the corresponding time. At each studied time, the figures are mapped at the same scale of colors. Consequently, it can be clearly seen that the model $_{1}$ based prediction results are highly comparable with those obtained by the models of model ${ }_{2}^{t}$. More importantly, the demonstrations of the entire room once again confirm that the heat is higher in the working time, especially in the afternoon, when there are happening of occupants activities. It can be observed that the cubicle area under occupancy is really hot of the temperature of above $24^{\circ} \mathrm{C}$. Nevertheless, the upper left corners of the figures demonstrate approximately steady and high temperature over time, which is owing to workings of the servers located in this area. Furthermore, except the server region, the areas close to main and back doors are always hotter than the others during the day. These phenomena can be understandable when insulation of the doors is weak.

Similarly, the prediction standard errors in the whole room at three different times mentioned above are mapped as demonstrated in figures $3 \mathrm{~d}, 3 \mathrm{e}$ and $3 \mathrm{f}$. Corresponding to the predicted mean demonstrations, the standard errors are also predicted by the model model $_{1}$ and the models model ${ }_{2}^{t}$, respectively. Generally, the predicted standard errors in the top row of figures $3 \mathrm{~d}, 3 \mathrm{e}$ and $3 \mathrm{f}$, which are obtained by the use of the model $_{1}$, are considerably smaller than those in the bottom row of these figures, which are obtained by each model model $t_{2}^{t}$ at corresponding time. Note that the comparisons are based on every pair of the predicted standard errors obtained by the two types of models at every individual location in the room. The premise behind these differences is that the spatio-temporal model model $_{1}$ consists of temporal elements in its forms. In other words, the spacetime model has temporal correlations of the compared point with the point before and the point after. This type of the correlation does not exist in the purely spatial model model ${ }_{2}^{t}$. It efficiently and effectively shows that the space-time model proposed in this work can have better predictions of spatiotemporal fields than spatial models.

\section{CONCLUSIONS}

An efficient approach of monitoring indoor climates in smart buildings was proposed, which involves modeling and predicting environmental fields in the building spaces. The indoor spatio-temporal environmental parameters are first modeled by Gaussian processes, which allows building management systems to observe the indoor climates at any time and at any locations via monitoring maps/surfaces of the environmental fields. From this, control laws/strategies for managing the building are then effectively designed to decline energy usage and grow occupant comfort in the building spaces. The proposed method was comprehensively implemented and then evaluated in a real space in a university building. The obtained results have demonstrated the efficiency of the approach.

\section{REFERENCES}

[1] B. Sun, P. B. Luh, Q. S. Jia, Z. Jiang, F.Wang, and C. Song, "Building energy management: Integrated control of active and passive heating, cooling, lighting, shading, and ventilation systems," IEEE Transactions on Automation Science and Engineering, vol. 10(3), pp. 588-602, 2013

[2] Commercial Buildings Energy Consumption Survey, U.S. Energy Information Adminstration, 2011.

[3] Building Energy Data Book, U.S. Department of Energy, 2011.

[4] D. Brunelli, I. Minakov, R. Passerone, and M. Rossi, "Smart monitoring for sustainable and energy-efficient buildings: A case study," in Proc. IEEE Workshop on Environmental, Energy and Structural Monitoring Systems, Trento, Italy, July 2015.

[5] L. D. Pereira, D. Raimondo, S. P. Corgnati, and M. G. Silva, "Assessment of indoor air quality and thermal comfort in Portuguese secondary classrooms: Methodology and results," Building and Environment, vol. 81, pp. 69-80, 2014

[6] D. Brunelli, I. Minakov, R. Passerone, and M. Rossi, "POVOMON: An ad-hoc wireless sensor network for indoor environmental monitoring," in Proc. IEEE Workshop on Environmental Energy and Structural Monitoring Systems, Naples, Italy, September 2014, pp. 1-6.

[7] Q. Zhu, J. Yi, S. Sheng, C. Wen, and H. Hu, "A computer-aided modeling and measurement system for environmental thermal comfort sensing," IEEE Transactions on Instrumentation and Measurement, vol. 64(2), pp. 478-486, 2015.

[8] C. Lin, "Forecasting indoor environment using ensemble-based data assimilation algorithms," Ph.D. dissertation, Concordia University, Montreal, Quebec, Canada, 2014.

[9] J. A. Burns, J. Borggaard, E. Cliff, and L. Zietsman, "An optimal control approach to sensor / actuator placement for optimal control of high performance buildings," in Proc. International Conference on High Performance Buildings, Purdue, USA, July 2012, pp. 1-7.

[10] G. Lowry and M. Lee, "Modelling the passive thermal response of a building using sparse BMS data," Applied Energy, vol. 78(1), pp. 53-62, 2004

[11] N. Cressie and C. K. Wikle, Statistics for spatio-temporal data. Wiley, 2011.

[12] N. A. Cressie, Statistics for spatial data. Wiley, 1991. 